\title{
Using $u$ band emission to trace star formation rates of galaxies
}

\author{
Zhimin Zhou \\ Key Laboratory of Optical Astronomy, National Astronomical Observatories, Chinese \\ Academy of Sciences, Beijing, 100012, China \\ email: zmzhou@bao.ac.cn
}

\begin{abstract}
Star formation rate (SFR) is one of the most important diagnostics in understanding the evolution of galaxies across cosmic times. In order to explore the possibility of using the optical $u$-band luminosities to estimate SFRs of galaxies, we show the correlations between $u$ band, $\mathrm{H} \alpha$ and infrared luminosities by combing the data from the South Galactic Cap $u$ band Sky Survey (SCUSS) with the Sloan Digital Sky Survey (SDSS) and the Wide-field Infrared Survey Explorer (WISE). We derive the $u$ versus $\mathrm{H} \alpha$ relation and the $u$ and $12 \mu \mathrm{m}$ relations to calibrate the $u$-band luminosity as an SFR indicator.
\end{abstract}

Keywords. galaxies: star formation, galaxies: ISM

\section{Introduction}

It is well-known that star formation is the critical activity to build up the stellar mass of galaxies. The star formation rate (SFR) is one of the most important diagnostics in understanding the evolution of galaxies. The measurement of the SFRs can give clues to the physical processes in shaping the galaxies. SFRs can be traced by many indicators, including both continuum and nebular recombination emission lines (Kennicutt \& Evans 2012), such as the ultraviolet (UV) continuum, $\mathrm{H} \alpha$ nebular line, infrared (IR) emission (Kennicutt 1998).

The optical $u$-band luminosity of a galaxy is dominated by young stars of age $<1$ Gyr, and has been proved to be a reasonable star formation tracer (Hopkins et al. 2003). The availability of recent and upcoming deep optical surveys, such as the Canada-FranceHawaii Telescope Legacy Survey (CFHTLS), the South Galactic Cap $u$ band Sky Survey (SCUSS; Zhou et al. 2016), the Large Synoptic Survey Telescope (LSST), provide an opportunity to measure SFRs of unprecedented large samples with $u$ band data. With the advent of these deep and wide-field surveys, it becomes necessary to recalibrate the $u$-band SFR.

\section{Data and Sample}

We investigate the $u$ band observations from SCUSS as an indicator of SFRs here in an effort to determine its feasibility and precision. SCUSS is a $u$ band $(\lambda \sim 3538 \AA)$ survey imaging $\sim 5,000 \mathrm{deg}^{2}$ in the northern part of the Southern Galactic Cap, it provides deeper $u$ band photometric data than SDSS. These data are used to compare with H $\alpha$ and IR SFR indicators to derive an accurate $u$-SFR calibration for normal star-forming galaxies. Holuminosities are derived from the emission line measurements of galaxy spectra from MPA-JHU spectroscopic analysis (Tremonti et al. 2004). IR luminosities are obtained from the ALLWISE source catalogue which provies positional and photometric information for over 747 million objects detected on the deep, coadded WISE Images. 

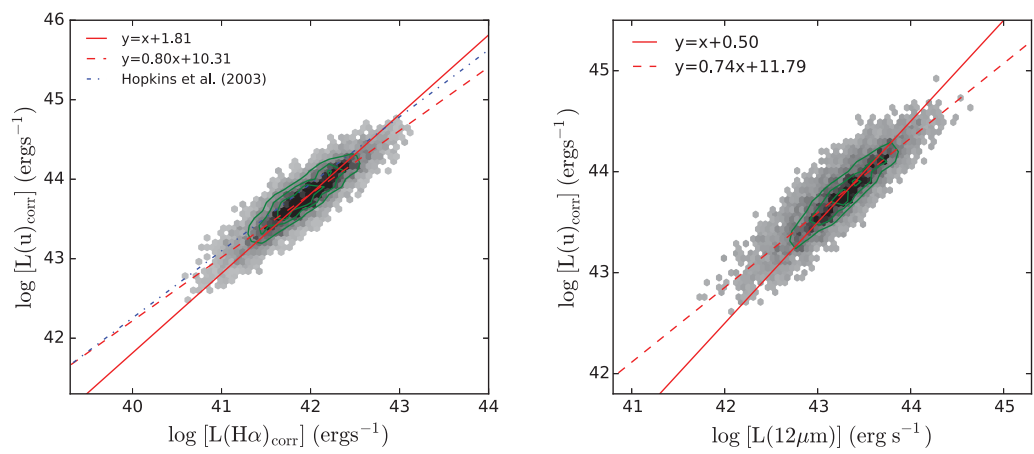

Figure 1. Left: Comparison of the luminosities of $u$ band and $\mathrm{H} \alpha$. The Holuminosities are derived from the MPA-JHU catalogue and are corrected for the dust extinction and aperture bias. The $u$ band luminosities are also corrected for the dust extinction. The green contours are number densities of the sample distribution. The red solid and dash lines represent the best linear and nonlinear fits in this work. The blue dash-dot line represents the relation by Hopkins et al. (2003) using SDSS DR1. Right: $u$-band luminosities as a function of the WISE $12 \mu \mathrm{m}$ luminosities. The $u$-band luminosities are corrected for the dust extinction. The best linear and nonlinear fittings are shown with solid and dashed lines, respectively.

We match the SCUSS sources with the MPA-JHU catalogue and ALLWISE catalogue. In order to produce the accurate SFR calibration, we select the pure star forming galaxies with no contamination of active galactic nucleus (AGN) based on the BPT diagnostic diagram (Baldwin et al. 1981).

\section{Result and Discussion}

Figure 1 compares the luminosities of $u$ band and H $\alpha$ emission line. It can be clearly seen that the attenuation-corrected $u$-band luminosities correlate tightly with the Balmer decrement-corrected H $\alpha$ luminosities with the Spearman rank-order correlation coefficient of 0.88 . The best linear and nonlinear fits are also plotted in the figure along with the relation from Hopkins et al. (2003) for comparison. Our fitting results are consistent to that of Hopkins et al. (2003). The relation of $u$ band and WISE $12 \mu \mathrm{m}$ luminosities is also shown in Figure 1 along with the linear and nonlinear fits plotted. The scatter of the nonlinear fits is 0.18 dex. The systematic residuals of our calibrations are mainly affected by dust attenuation and old stellar population.

\section{References}

Baldwin J. A., Phillips M. M., Terlevich R., 1981, PASP, 93, 5

Kennicutt, R. C. 1998, ARAA, 36, 189

Kennicutt, R. C. \& Evans, N. J. 2012, ARAA, 50, 531

Hopkins, A. M., Miller, C. J., Nichol, R. C., et al. 2003, ApJ, 599, 971

Tremonti, C. A., Heckman, T. M., Kauffmann, G., et al. 2004, ApJ, 613, 898

Zhou, X., Fan, X.-H., Fan, Z., et al. 2016, Research in Astronomy and Astrophysics, 16, 69 